\title{
Ready for the sixth year of Internal and Emergency Medicine
}

\author{
Domenico Prisco
}

(C) SIMI 2011

Dear Readers,

With this issue IEM starts its sixth year of life. For those who have worked in this project, it is a great pleasure to see the continuous growth of our creature.

After the successful obtaining of the first impact factor (IF $2009=2.371$ ) we all continue to work to increase the quality of the papers published and the pleasure of the readers to have nice and useful articles. Our journal is now internationally recognized as an important one in our academic field.

Much work still needs to be done. The section of Clinical evidence and Health Technology Assessment leaded by the past Editor-in-chief Gian Franco Gensini with the invaluable work of Lorenzo Moja and Gianni Virgili (among the others) is growing. The Emergency section, leaded by my co-editor Peter Rosen, with Kevin Ban and a group of distinguished experts of different countries, receives many submissions and our colleagues do a great job. Also the Internal Medicine section has an increased number of submissions and several members of Editorial Board have begun to directly manage some papers as editors.

All the work of IEM, as a whole, has much increased during the second half of 2010, because of the announcement of Impact Factor. We have received over 250 new submissions versus 120 in the first part of the year. Thus, the submission rate is over $+100 \%$ in the second part of the year versus the first and $+60 \%$ in the all 2010 versus 2009.

D. Prisco $(\bowtie)$

Dipartimento di Area Critica Medico Chirurgica, Università degli Studi di Firenze, Viale Morgagni 85, 50134 Florence, Italy

e-mail: priscod@aou-careggi.toscana.it
This means, on the other hand, that, more or less, the mean rejection rate should be $70-75 \%$ at the moment.

Thus, there has been more work for all the members of Editorial board, Advisory board and Operative group that again I thank for their invaluable work together with all people from Springer.

Usage of IEM articles increased by 19\% from 2008 to 2009. Moreover, in the period January-August, the 2010 growth rate of full-text downloads reached $36 \%$.

PubMed linkouts number represents the number of links from PubMed to SpringerLink full-texts. The more the linkouts are, the more the visibility of the journal grows. Comparing the data related to the period January-August, PubMed linkouts increased by 21\% from 2009 to 2010.

A special thank to all reviewers who have contributed to the success of our Journal, for the time and dedication spent. We have still the problem of those colleagues who do not reply after having been invited to act as reviewers, which produces delays in the process of reviewing. Again we ask potential reviewers to help us at least with a prompt reply and with the suggestion of other potential reviewers when they cannot do the job.

As I previously wrote, we encourage our members to provide feedback on the Journal and its content. If there are suggestions, please let us know.

I am sure you will continue to support our Journal and I wish you all a wonderful 2011!

Domenico Prisco

Editor-in-Chief

Internal and Emergency Medicine 